\title{
一类含有自然数最小素因子的和 *
}

\author{
曹惠中
}

(山东大学数学系, 济南 250100 )

\section{关链词最小因子、最大萧因子、可加函数}

令 $P(n), Q(n)$ 分别表示自然数 $n$ 的最小素因子和最大素因子. 近年来, Erdös, Ivić 等学 者对含有 $P(n), Q(n)$ 的倒数的和进行了研究. 例如 Ivic ${ }^{[1]}$ 证明了

$$
\begin{aligned}
& \sum_{2 \leqslant n \leqslant x} \frac{\Omega(n)-\omega(n)}{Q(n)}=\left\{\sum_{p} \frac{1}{p^{2}-p}+O\left(\left(\frac{\log \operatorname{lng} x}{\log x}\right)^{\frac{1}{2}}\right)\right\}_{2 \leqslant n \leqslant x} \frac{1}{Q(n)}, \\
& \sum_{2 \leqslant n \leqslant x} \frac{\omega(n)}{Q(n)}=\left\{\left(\frac{2 \log x}{\log \log x}\right)^{\frac{1}{2}}\left(1+O\left(\frac{\log \log \log x}{\log \log x}\right)\right)\right\}_{2 \leqslant n \leqslant x} \frac{1}{Q(n)},
\end{aligned}
$$

本文和通常一样, 令 $\omega(n), \Omega(n)$ 分别表示 $n$ 的不同素因子的个数和全部素因子的个数. 张文 鹏 ${ }^{[2]}$ 证明了

$$
\begin{aligned}
\sum_{2 \leqslant n \leqslant x} \frac{\omega(n)}{p(n)} & =c_{0} x \log \log x+O(x), \\
\sum_{2 \leqslant n \leqslant x} \frac{\Omega(n)}{p(n)} & =c_{0} x \log \log x+O(x),
\end{aligned}
$$

本文设 $c_{0}=\sum_{p} \frac{w(p)}{p^{2}}, w(p)=\prod_{p_{1}<p}\left(1-\frac{1}{p_{1}}\right), p>2, w(2)=1$ 及 $p, p_{1}$ 表示素数.

设 $h(n)$ 是满足 $h(o)=0$ 的任一数论函数, 当 $n=\prod_{i=1}^{s} p_{i}^{\beta_{i}}$ 时, 定义 $H(n)=\sum_{i=1}^{s} h\left(\beta_{i}\right)$. 并定义 $H(1)$ $=0$ (见文哉[3]). $H(n)$ 是一类可加函数的抽象, 例如, 若 $h(n)$ 分别取 $1, n, \log (1+n), \frac{n}{n+1}$ 及 $\frac{n}{2}$, 则相应的 $H(n)$ 分别为 $\omega(n), \Omega(n), \log d(n), \frac{1}{d(n)} \sum_{d \mid n} \omega(d)$ 及 $\frac{1}{d(n)} \sum_{d \mid n} \Omega(d)$, 此处 $d(n)$ 是除数函数. 注意上面所列举的 $h(n)$ 都满足 $h(n) \leqslant n$. 本文的目的是证明

定理 若 $h(n)=O\left(n^{k}\right), k>0$, 则

$$
\sum_{2 \leqslant n \leqslant x} \frac{H(n)}{p(n)}=c_{0} h(1) x \log \log x+\left(c_{1} h(1)+c_{2}+c_{3}\right) x+O\left(\frac{x}{\log x}\right),
$$

1993-05-27 收稿.

*国家自然科学基金资助项目. 
此处 $c_{1}=\sum_{p} \frac{w(p)}{p^{2}}\left(1+B-\sum_{p_{1} \leqslant p} \frac{1}{p_{1}}\right), B=\gamma+\sum_{p}\left\{\frac{1}{p}+\log \left(1-\frac{1}{p}\right)\right\}$ 及 $\gamma$ 为 Euler 常数, $c_{2}=$ $\sum_{p} \frac{w(p)}{p} \sum_{m=2}^{\infty}\{h(m)-h(m-1)\} p^{-m}$ 及 $c_{3}=\sum_{p} \frac{w(p)}{p^{2}} \sum_{p_{1}>p} \sum_{m=2}^{\infty}\{h(m)-h(m-1)\} p_{1}^{-m}$.

为了证明定理需要下述的引理.

引理 1 令 $\varphi(p, x)=\sum_{n \leqslant x} 1$. 若 $p \leqslant x$, 则

$$
p(n) \geqslant p
$$

$$
\varphi(p, x)=w(p) x+o\left\{x(\log p)^{\frac{5}{2}} \exp \left(-\frac{\log x}{3 \log p}\right)\right\} .
$$

证 当 $\operatorname{Re} s>1$, 有

$$
1+\sum_{\substack{n=1 \\ p(n) \geqslant p}}^{\infty} \frac{1}{n^{s}}=\prod_{p_{1} \geqslant p}\left(1-\frac{1}{p_{1}^{s}}\right)^{-1}=\zeta(s) \prod_{p_{1}<p}\left(1-\frac{1}{p_{1}^{s}}\right) .
$$

因此当 $x$ 取半整数并在文献[4] 的第六章第五节的定理 2 中取 $b=1+\frac{1}{\log x}$ 时,有

$$
1+\varphi(p, x)=\frac{1}{2 \pi i} \int_{b-i T}^{b+i T} \zeta(s) \prod_{p_{1}<p}\left(1-\frac{1}{p_{1}^{s}}\right) \frac{x^{s}}{s} \mathrm{~d} s+O\left(\frac{x \log x}{T}\right) .
$$

令 $c=1-\left(\frac{1}{3}+\frac{1}{100}\right) \frac{1}{\log p}$. 则当 $\operatorname{Res} \geqslant c$ 时, 有

$$
\begin{gathered}
\left|\prod_{p_{1}<p}\left(1-\frac{1}{p_{1}^{s}}\right)\right| \leqslant \exp \left(\sum_{p_{1}<p} \frac{1}{p_{1}^{c}}\right) \leqslant \exp \left\{e^{\left(\frac{1}{3}+\frac{1}{100}\right)} \sum_{p_{1}<p} \frac{1}{p_{1}}\right\} \\
\leqslant \exp \left\{\frac{3}{2}(\log \log p+O(1))\right\} \ll(\log p)^{\frac{3}{2}} .
\end{gathered}
$$

将 (1) 式中的积分路线平移至 Res $=c$ 处, 由留数定理及 (2) 式即得

$$
\begin{aligned}
1+ & \varphi(p, x)=w(p) x+o\left\{(\log p)^{\frac{3}{2}} x^{c} \int_{o}^{T} \frac{|\zeta(c+i t)|}{t+1} \mathrm{~d} t\right\} \\
& +O\left\{(\log p)^{\frac{3}{2}} \int_{c}^{b}|\zeta(\sigma+i T)| \frac{x^{\sigma}}{T} \mathrm{~d} \sigma\right\}+O\left(\frac{x \log x}{T}\right) .
\end{aligned}
$$

由 Riemann-Zata 函数的熟知结果, 注意到 $c>\frac{1}{2}$, 有

$$
\int_{0}^{T} \frac{|\zeta(c+i t)|}{t+1} \mathrm{~d} t \leqslant\left(\int_{0}^{T} \frac{|\zeta(c+i t)|^{2}}{t+1} \mathrm{~d} t \int_{0}^{T} \frac{\mathrm{d} t}{t+1}\right)^{\frac{1}{2}} \ll \log T
$$

及 


$$
\int_{c}^{b}|\zeta(\sigma+i T)| x^{\sigma} \mathrm{d} \sigma \ll \log x \int_{c}^{b} T^{\frac{1}{2} \cdot(1-\sigma)} x^{\sigma} \mathrm{d} \sigma=\sqrt{T} \log x \int_{c}^{b}\left(\frac{x}{\sqrt{T}}\right)^{\sigma} \mathrm{d} \sigma \ll x \log x .
$$

取 $T=\frac{\log x}{\log p} \exp \left(\frac{\log x}{3 \log p}\right)$, 由 (3)-(5) 式即得引理.

引理 $2 \sum_{2 \leqslant n \leqslant x} \frac{\omega(n)}{p(n)}=c_{0} x \log \log x+c_{1} x+O\left(\frac{x}{\log x}\right)$, 此处 $c_{1}=\sum_{p} \frac{w(p)}{p^{2}}\left(1+B-\sum_{p_{1} \leqslant p}\right.$ $\left.\frac{1}{p_{1}}\right), B=\gamma+\sum_{p}\left\{\frac{1}{p}+\log \left(1-\frac{1}{p}\right)\right\}$ 及 $\gamma$ 为 Euler 常数.

证

$$
\begin{aligned}
\sum_{2 \leqslant n \leqslant x} \frac{\omega(n)}{p(n)} & =\sum_{p \leqslant x} \frac{1}{p} \sum_{\substack{n \leqslant x \\
p(n)=p}} \omega(n) \\
& =\sum_{p \leqslant x} \frac{1}{p} \sum_{\substack{m \leqslant x / p \\
p(m) \geqslant p}} \omega(p m)+\sum_{p \leqslant x} \frac{1}{p} \\
& =\sum_{p \leqslant x} \frac{1}{p} \sum_{\substack{m \leqslant x / p \\
p(m) \geqslant p}} \omega(m)+\sum_{p \leqslant x} \frac{1}{p} \sum_{\substack{m \leqslant x / p \\
p(m)>p}} 1+O(\log \log x) \\
& =S_{1}+S_{2}+O(\log \log x) .
\end{aligned}
$$

注意到 $\sum_{p \leqslant y} \frac{1}{p}=\log \log y+B+O\left(\frac{1}{\log y}\right)$, 由引理 1 可得

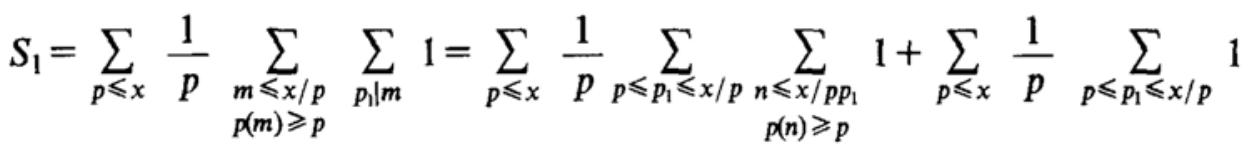

$$
\begin{aligned}
& =\sum_{p \leqslant \sqrt[3]{x}} \frac{1}{p} \sum_{p \leqslant p_{1} \leqslant x / p^{2}} \sum_{\substack{n \leqslant x) p p_{1} \\
p(n) \geqslant p}} 1+O\left(\sum_{p \leqslant \sqrt{x}} \frac{1}{p} \pi\left(\frac{x}{p}\right)\right) \\
& =\sum_{p \leqslant \sqrt[3]{x}} \frac{1}{p} \sum_{p \leqslant p_{1} \leqslant x / p^{2}}\left\{\frac{x}{p p_{1}} w(p)+O\left(x \frac{1}{p p_{1}}(\log p)^{\frac{5}{2}} \exp \left(-\frac{\log x / p_{1}}{3 \log p}\right)\right)\right\}+O\left(\frac{x}{\log x}\right) \\
& =x \sum_{p \leqslant \sqrt[3]{x}} \frac{w(p)}{p^{2}} \sum_{p_{1} \leqslant x / p^{2}} \frac{1}{p_{1}}-x \sum_{p \leqslant \sqrt[3]{x}} \frac{w(p)}{p^{2}} \sum_{p_{1}<p} \frac{1}{p_{1}} \\
& +O\left\{x \sum_{p \leqslant \sqrt[3]{x}} \frac{1}{p^{2}}(\log p)^{\frac{5}{2}} \exp \left(-\frac{\log x}{3 \log p}\right) \sum_{p_{1} \leqslant x / p^{2}} p_{1}^{\frac{1}{3 \log p}-1}\right\}+O\left(\frac{x}{\log x}\right) \\
& =x \sum_{p \leqslant \sqrt[3]{x}} \frac{w(p)}{p^{2}}\left\{\log \log \frac{x}{p^{2}}+B+O\left(\frac{1}{\log x}\right)\right\}-x \sum_{p} \frac{w(p)}{p^{2}} \sum_{p_{1}<p} \frac{1}{p_{1}} \\
& +O\left\{\frac{x}{\log x} \sum_{p \leqslant \sqrt[3]{x}} \frac{1}{p^{2}}(\log p)^{\frac{7}{2}} \exp \left(-\frac{\log x}{3 \log p}\right)\left(\frac{x}{p^{2}}\right)^{\frac{1}{3 \log p}}+\sum_{p \leqslant \sqrt[3]{x}} \frac{x}{p^{2}}(\log p)^{\frac{7}{2}}\right. \\
& \left.\exp \left(-\frac{\log x}{3 \log p}\right)\left(\frac{x}{p^{2}}\right)^{\frac{1}{6 \log p}}\right\}+O\left(\frac{x}{\log x}\right)
\end{aligned}
$$




$$
=c_{0} x \log \log x+x \sum_{p} \frac{w(p)}{p^{2}}\left(B-\sum_{p_{1}<p} \frac{1}{p_{1}}\right)+O\left(\frac{x}{\log x}\right),
$$

此处用到当 $-1<\beta<0$ ( $\beta$ 与 $x$ 有关) 时, 有

$$
\begin{gathered}
\sum_{p \leqslant x} p^{\beta=} \int_{2^{-}}^{x} t^{\beta} \mathrm{d} \pi(t) \ll \frac{x^{\beta+1}}{\log x}+\left(\int_{2}^{\sqrt{x}}+\int_{\sqrt{x}}^{x}\right) \frac{t^{\beta}}{\log t} \mathrm{~d} t \\
\ll \frac{1}{1+\beta} \frac{x^{1+\beta}}{\log x}+\frac{1}{1+\beta} x^{\frac{1+\beta}{2}} .
\end{gathered}
$$

本文用 $p^{\prime}$ 表示大于 $p$ 的最小素数, 则 $p<p^{\prime}<2 p$. 再由引理 1 , 有

$$
\begin{aligned}
S_{2} & =\sum_{p p^{\prime} \leqslant x} \frac{1}{p} \sum_{\substack{m \leqslant x / p \\
p(m) \geqslant p^{\prime}}} 1=x \sum_{p p^{\prime} \leqslant x} \frac{w\left(p^{\prime}\right)}{p^{2}}+O\left\{x \sum_{p p^{\prime} \leqslant x} \frac{1}{p^{2}}(\log p)^{\frac{5}{2}} \exp \left(-\frac{\log x}{3 \log 2 p}\right)\right\} \\
& =x \sum_{p} \frac{w\left(p^{\prime}\right)}{p^{2}}+O\left(x \sum_{p>\sqrt{\frac{x}{2}}} \frac{1}{p^{2}}\right)+O\left\{x \sum_{p \leqslant \sqrt{x}} \frac{1}{p^{2}}(\log p)^{\frac{5}{2}} \exp \left(-\frac{\log x}{3 \log 2 p}\right)\right\} \\
& =x \sum_{p} \frac{w(p)}{p^{2}}\left(1-\frac{1}{p}\right)+O\left(\frac{x}{\log x}\right) .
\end{aligned}
$$

由 (6)-(8) 式即得引理 2 .

定理的证明 注意到 $h(o)=0$, 有

$$
\begin{aligned}
\sum_{2 \leqslant n \leqslant x} \frac{H(n)}{p(n)} & =\sum_{p \leqslant x} \frac{1}{p} \sum_{\substack{n \leqslant x \\
p(n)=p}} \sum_{p_{1}^{m} \mid n}\{h(m)-h(m-1)\} \\
& =h(1) \sum_{p \leqslant x} \frac{1}{p} \sum_{\substack{n \leqslant x \\
p(n)=p}} \omega(n)+\sum_{p \leqslant x} \frac{1}{p} \sum_{\substack{n \leqslant x \\
p(n)=p}} \sum_{\substack{p_{1}^{m} \mid n \\
m \geqslant 2}}\{h(m)-h(m-1)\} \\
& =h(1) \sum_{2 \leqslant n \leqslant x} \frac{\omega(n)}{p(n)}+S_{3} .
\end{aligned}
$$

现令

$$
g(n)=\left\{\begin{array}{l}
1, \text { 当 } n=p^{m}, m \geqslant 2 ; \\
0, \text { 此外. }
\end{array}, G(n)=\left\{\begin{array}{l}
h(m)-h(m-1), \text { 当 } n=p^{m}, m \geqslant 2 ； \\
0, \text { 此外. }
\end{array}\right.\right.
$$

从而 $S_{3}$ 可改写为

$$
\begin{aligned}
S_{3}= & \sum_{p \leqslant x} \frac{1}{p} \sum_{\substack{d s \leqslant x \\
p(d s)=p}} g(d) G(d)=\sum_{p \leqslant x} \frac{1}{p} \sum_{\substack{d s \leqslant x \\
p(d)=p(s)=p}} g(d) G(d) \\
& +\sum_{p \leqslant x} \frac{1}{p} \sum_{\substack{d s \leqslant x \\
p(d)=p, p(s)>p}} g(d) G(d)+\sum_{p \leqslant x} \frac{1}{p} \sum_{\substack{d(d) \leqslant x \\
p(d)>p, p(s)=p}} g(d) G(d) \\
& +O\left(\sum_{p \leqslant x} \frac{1}{p} \sum_{p^{m} \leqslant x, m \geqslant 2} m^{k}\right)=S_{4}+S_{5}+S_{6}+O\left(\log ^{k+2} x\right) .
\end{aligned}
$$

以下分别估计 $S_{i}, 4 \leqslant i \leqslant 6$. 
令 $m^{k}=O\left(\left(\frac{5}{4}\right)^{m}\right)$, 注意到 $\frac{5}{8} e^{\frac{1}{3}}<1$ 及 $\frac{\log \frac{5}{4}}{\log 2}<\frac{1}{2}$, 由引理 1 可得

$$
\begin{aligned}
& S_{4}=\sum_{p \leqslant x} \frac{1}{p} \sum_{\substack{d \leqslant x \\
p(d)=p}} \sum_{\substack{s \leqslant x / d \\
p(s)=p}} g(d) G(d)=\sum_{p \leqslant \sqrt{x}} \frac{1}{p} \sum_{\substack{d \leqslant x / p \\
p(d)=p}} g(d) G(d) \sum_{\substack{s \leqslant x / d \\
p(s)=p, p \neq s}} 1 \\
& +\sum_{p \leqslant \sqrt{x}} \frac{1}{p} \sum_{\substack{d \leqslant x / p \\
p(d)=p}} g(d) G(d)=\sum_{p \leqslant \sqrt[3]{x}} \frac{1}{p} \sum_{\substack{d \leqslant x / p^{2} \\
p(d)=p}} g(d) G(d) \sum_{\substack{l \leqslant x / d p \\
p(l) \geqslant p}} 1+O\left(\log x^{k+2}\right) \\
& =x \sum_{p \leqslant \sqrt[3]{x}} \frac{w(p)}{p^{2}} \sum_{p^{m} \leqslant x / p^{2}}\{h(m)-h(m-1)\} p^{-m}+O\left\{x \sum_{p \leqslant \sqrt[3]{x}} \frac{1}{p^{2}}(\log p)^{\frac{5}{2}}\right. \\
& \left.\exp \left(-\frac{\log x}{3 \log p}\right) \sum_{m=2}^{\infty}\left(\frac{5}{4 p} e^{\frac{1}{3}}\right)^{m}\right\}+O\left(\log x^{k+2}\right) \\
& =x \sum_{p \leqslant \sqrt[3]{x}} \frac{w(p)}{p^{2}} \sum_{m=2}^{\infty}\{h(m)-h(m-1)\} p^{-m}+O\left\{x \sum_{p \leqslant \sqrt[3]{x}} \frac{w(p)}{p^{2}} \sum_{m>\frac{\log x}{\log p}}\left(\frac{5}{4 p}\right)^{m}\right\}+O\left(\frac{x}{\log x}\right) \\
& =x \sum_{p} \frac{w(p)}{p^{2}} \sum_{m=2}^{\infty}\{h(m)-h(m-1)\} p^{-m}+O\left(\frac{x}{\log x}\right),
\end{aligned}
$$

因为

$$
x \sum_{p \leqslant \sqrt[3]{x}} \frac{w(p)}{p^{2}} \sum_{m>\frac{\log x}{\log p}-2}\left(\frac{5}{4 p}\right)^{m} \leqslant x \sum_{p \leqslant \sqrt[3]{x}} \frac{w(p)}{p^{2}}\left(\frac{5}{4 p}\right)^{\frac{\log x}{\log p}-2} \ll x^{\frac{\log \frac{5}{4}}{\log 2}+\frac{1}{3}} .
$$

由引理 1 , 同理可得

$$
\begin{aligned}
S_{5} & =\sum_{p \leqslant x} \frac{1}{p} \sum_{\substack{d \leqslant x \\
p(d)=p}} g(d) G(d) \sum_{\substack{s \leqslant x / d \\
p(s) \geqslant p^{\prime}}} 1 \\
& =\sum_{p \leqslant \sqrt{x}} \frac{1}{p} \sum_{\substack{d \leqslant x / p^{\prime} \\
p(d)=p}} g(d) G(d) \sum_{\substack{s \leqslant x / d \\
p(s) \geqslant p^{\prime}}} 1 \\
& =x \sum_{p} \frac{w(p)}{p}\left(1-\frac{1}{p}\right) \sum_{m=2}^{\infty}\{h(m)-h(m-1)\} p^{-m}+O\left(\frac{x}{\log x}\right) .
\end{aligned}
$$

再次使用引理 1 , 我们有

$$
\begin{aligned}
& S_{6}=\sum_{p \leqslant x} \frac{1}{p} \sum_{\substack{d \leqslant x \\
p(d)>p}} g(d) G(d) \sum_{\substack{s \leqslant x / d \\
p(s)=p}} 1=\sum_{p \leqslant \sqrt{x}} \frac{1}{p} \sum_{\substack{d \leqslant x / p \\
p(d)>p}} g(d) G(d) \sum_{\substack{s \leqslant x / d \\
p(s)=p, s \neq p}} 1 \\
& +\sum_{p \leqslant \sqrt{x}} \frac{1}{p} \sum_{\substack{d \leqslant x / p \\
p(d)>p}} g(d) G(d)=\sum_{p \leqslant \sqrt[3]{x}} \frac{1}{p} \sum_{\substack{d \leqslant x / p^{2} \\
p(d)>p}} g(d) G(d) \sum_{\substack{l \leqslant x / d p \\
p(l) \geqslant p}} 1
\end{aligned}
$$




$$
\begin{aligned}
& +O\left(\sum_{p \leqslant \sqrt{x}} \frac{1}{p} \sum_{\substack{p_{1}^{m} \leqslant x \\
m \geqslant 2, p_{1}>p}} m^{k}\right)=x \sum_{p \leqslant \sqrt[3]{x}} \frac{w(p)}{p^{2}} \sum_{\substack{p_{1}^{m} \leqslant x / p^{2} \\
m \geqslant 2, p_{1}>p}}\{h(m)-h(m-1)\} p_{1}^{-m} \\
& +O\left\{x \sum_{p \leqslant \sqrt[3]{x}} \frac{1}{p^{2}}(\log p)^{\frac{5}{2}} \exp \left(-\frac{\log x}{3 \log p}\right) \sum_{\substack{p_{1}^{m} \leqslant x \\
m \geqslant 2, p_{1}>p}} m^{k} p_{1}^{-m} \exp \left(\frac{m \log p_{1}}{3 \log p}\right)\right\} \\
& +O\left(\sum_{p \leqslant \sqrt[3]{x}} \frac{1}{p} \sum_{p<p_{1} \leqslant \sqrt{x}} \sum_{2 \leqslant m \leqslant \frac{\log x}{\log p_{1}}} m^{\mathrm{k}}\right) \\
& =x \sum_{p \leqslant \sqrt[3]{x}} \frac{w(p)}{p^{2}} \sum_{p<p_{1} \leqslant \sqrt{x} / p} \sum_{m=2}^{\infty}\{h(m)-h(m-1)\} p_{1}^{-m}+O\left\{x \sum_{p \leqslant \sqrt[3]{x}} \frac{w(p)}{p^{2}} \sum_{p<p_{1} \leqslant \sqrt{x} / p}\right. \\
& \left.\sum_{m>\frac{\log x}{\log p_{1}}} \sum_{-2 \frac{\log p}{\log p_{1}}}\left(\frac{5}{4 p_{1}}\right)^{m}\right\}+O\left\{x \sum_{p \leqslant \sqrt[3]{x}} \frac{1}{p^{2}}(\log p)^{\frac{5}{2}} \exp \left(-\frac{\log x}{3 \log p}\right) \sum_{2 \leqslant m \leqslant \log x}\right. \\
& \left.m^{k} \sum_{p_{1} \leqslant \sqrt{x}} \frac{1}{p_{1}^{2\left(1-\frac{1}{3 \log 2}\right)}}\right\}+O\left(\sqrt{x}(\log x)^{k+1}\right)=x \sum_{p \leqslant \sqrt[3]{x}} \frac{w(p)}{p^{2}} \sum_{p_{1}>p} \sum_{m=2}^{\infty}\{h(m)-h(m-1)\} p_{1}^{-m} \\
& +O\left(x \sum_{p \leqslant \sqrt[3]{x}} \frac{w(p)}{p^{2}} \sum_{p_{1}>\sqrt{x} / p} \frac{1}{p_{1}^{2}}\right)+O\left\{x \sum_{p \leqslant \sqrt[3]{x}} \frac{1}{\log p} \sum_{p_{1} \leqslant \sqrt{x} / p}\left(\frac{5}{4 p_{1}}\right)^{\frac{\log x}{\log p_{1}}}\right\} \\
& +O\left(\frac{x}{\log x}\right)=c_{3} x+O\left(\frac{x}{\log x}\right) \text {. }
\end{aligned}
$$

从引理 2 及 (9)-(13) 式即得定理.

在定理中取 $h(n)=n$ 即得

及

$$
\sum_{2 \leqslant n \leqslant x} \frac{\Omega(n)}{p(n)}=c_{0} x \log \log x+\left(c_{1}+a_{0}\right) x+O\left(\frac{x}{\log x}\right)
$$

$$
\sum_{2 \leqslant n \leqslant x} \frac{\Omega(n)-\omega(n)}{p(n)}=a_{0} x+o\left(\frac{x}{\log x}\right)
$$

此处

$$
a_{0}=\sum_{p} \frac{w(p)}{p^{2}}\left\{\frac{1}{p}+\sum_{p_{1} \geqslant p} \frac{1}{p_{1}\left(p_{1}-1\right)}\right\} .
$$

\section{参考文献}

[1] Ivic, A., Acta Arith., 1987, 49:21-33.

[2] 张文成, 数学学报, $1989,32(2): 260-267$.

[3] Duncan, R. L., Duke Math. J., 1966, 33:507-510.

[4]潘承洞、潘承䞞,解析数论基础,科学出版社, 1991. 